-Original Article-

\title{
Assessment of Computerized Method for Correction of Optical Distortion of Facial Images
}

\author{
Hitoshi Biwasaka*1,*5, Takuya Tokuta*1,*5, Yoshitoshi Sasaki*1,*5, Takashi Takagi*2 \\ Toyohisa Tanijiri*3, Sachio Miyasaka*4, Masataka Takamiya*5 and Yasuhiro Aoki*5 \\ Forensic Science Laboratory, Iwate Prefectual Police H.Q.*1 \\ 8-10 Uchimaru, Morioka, Iwate 020-8540, Japan \\ Forensic Science Laboratory, Miyagi Prefectural Police H.Q.*2 \\ 3-8-1 Honchou, Aobaku, Sendai, Miyagi 980-8410, Japan \\ Medic Engineering Co.*3 \\ 11-1 Higashihirakichou, Takano, Sakyoku, Kyoto, 606-8107, Japan \\ National Research Institute of Police Science*4 \\ 6-3-1 Kashiwanoha, Kashiwa, Chiba 277-0882, Japan \\ Department of Legal Medicine, Iwate Medical University School of Medicine*5 \\ 19-1 Uchimaru, Morioka, Iwate 020-8505, Japan
}

(Received 3 August 2007; accepted 12 October 2007)

The availability of software developed to correct the distortion associated with non-metric cameras was examined using a test chart composed of 63 gridiron dots and two CCD cameras: a commercially available camera as the reference and an actual surveillance camera installed in a bank. The software was designed to calculate distortion parameters such as the radial and tangential distortion coefficients and the principal points. Constant parameter values were obtained when test charts were taken at six positions in the lens field. The average of displacement errors between the 63 dots on the corrected image and those on the presumed ideal image was measured. In the reference camera, a pre-correction error of $1.24 \mathrm{~mm}$ decreased to $0.08 \mathrm{~mm}$ after correction. In the surveillance camera the error decreased from $7.92 \mathrm{~mm}$ to $0.33 \mathrm{~mm}$. The present method was compared with two others: the graph method and the Open CV method. The former uses a distortion graph, which indicates the distortion level $(\%)$ at several locations from the center to the radial direction of the lens field. The latter is a free camera calibration software tool, developed by Intel Co. for the calculation of the intrinsic camera parameters of video camera lenses. The present method was associated with smaller error values than either of the other two methods. It is therefore suggested that this method could usefully be applied to distortion correction of facial images taken by surveillance cameras.

Key words: Facial image, Distortion, Surveillance camera, Computerized correction, Camera calibration 


\section{Introduction}

Two dimensional (2D) facial images taken by surveillance cameras are commonly used for the identification of suspects and are often admitted in court as direct evidence. The comparison of facial images taken with such cameras with identity photographs of suspects in custody is performed by noting the shape of facial outlines and components. However, differences in camera angles, in distortion due to the refractive index of the lens, and in the perspective due to the distance to the subject often result in discrepancies between facial images in crime scene photographs and those of suspects in custody. In order to resolve differences between facial images and to correct the perspective of three dimensional (3D) images, a new identification system for $2 \mathrm{D} / 3 \mathrm{D}$ facial superimposition has been developed ${ }^{1,2)}$. However, differences in magnification between the center and periphery of the lens field cause distortion effects such as pincushion and barrel images. We have previously examined methods of correcting distortion induced in images taken by the surveillance camera at an automated teller machine (ATM)..3) Although commercial software for distortion correction designed to straighten the curvature of linear subjects in images is available, it has not been confirmed whether or not the correction is carried out accurately over the entire field of the image. Because detailed estimation of the error between 2D facial images and 3D ones is required, such methods are unsuitable for personal identification $^{1,2)}$. Surveillance cameras commonly use low-cost, wide angle lenses, which generate considerable distortion. Because the distortion parameters (distortion coefficients, principal point, and focal length) of surveillance camera lenses are rarely shown in product specifications (unlike in the case of single lens reflex cameras), the distortion parameters must be measured directly from the camera lens itself. Distortion correction methods can be classified into two categories: photogrammetric calibration and self-calibration. In the former, distortion parameters are measured by taking different planes of the object such as a test chart $^{6-11)}$, which has recently been applied to images recognition for construction of 3D images from $2 \mathrm{D}$ images ${ }^{6,7)}$. In the latter, the leveling is performed by taking images of multiple aspects of a static object, a method which has been used in photographic measurements ${ }^{12-15)}$. Because surveillance cameras are usually placed in a fixed position, the former method, which does not require the camera to be moved, would be preferable. Recently, one of the present authors (Tanijiri) reported a practical method for the calibration of CCD cameras using a test chart image ${ }^{16)}$. In this study, we examined the utility of this method through the correction of images taken by a model camera and by an actual surveillance camera, set at ATM. The corrected images were compared with the undistorted (ideal) image. Furthermore, the present method was compared with the distortion graph provided by the manufacturer, and with the Open CV method ${ }^{17)}$.

\section{Camera}

\section{Materials and methods}

\subsection{Reference CCD camera and its lenses}

A CCD camera (CS8550DiF [Toshiba electronic industry, Japan]) was used as the reference. The scanning area of the CCD is 4.88 $(H) \times 3.66(V) \mathrm{mm}$ (Equivalent to $1 / 3$ type) and the output image is $640(H) \times 480(V)$ pixels. The interface specification is IEEE 1394. The lenses were the TAMRON 12 FM 45 (focal length $(\mathrm{F})=4.5 \mathrm{~mm})$, and the UPC H3616FI $(\mathrm{F}$ $=3.6 \mathrm{~mm})$. The output signals of the reference camera were fed into a PC (Latitude D505, Dell, Japan) via the PC card interface (Zenkuman ${ }^{\mathrm{TM}}$ CFW-46, Technoscope, Japan).

\subsection{Surveillance camera}

The surveillance camera (CCD: 1/3 type, YK2050, YOKO) had been in use at an ATM. The camera was equipped with a $3.7 \mathrm{~mm}$ lens and connected to the camera controller. The output image from the camera was $510(H) \times$ $492(V)$ pixels. The analog signals put out by the controller were converted to digital signals by a converter (ADVC 110, Canopus, Japan) and 
the digital signals were fed into a PC.

\section{The present method}

\subsection{Test chart}

The $9 \times 7$ black dot pattern was made by image processing software (HANAKO, Justsystem, Japan) and printed on a lusterless white board (Fig. 1).

\subsection{Main principle of the present method}

The model for the radial and decentering distortions is given by the following equations ${ }^{6,7)}$, in which $(\bar{u}, \bar{v})$ and $(u, v)$ are the ideal (distortion-free) and uncorrected (distorted) image coordinates, respectively:

$$
\begin{aligned}
\bar{u}= & u+\left(u-c_{x}\right)\left[k_{1} r^{2}+k_{2} r^{4}+2 p_{1} y\right. \\
& \left.+p_{2}\left(\frac{r^{2}}{x}+2 x\right)\right] \\
\bar{v}= & v+\left(v-c_{y}\right)\left[k_{1} r^{2}+k_{2} r^{4}+2 p_{2} x\right. \\
& \left.+p_{1}\left(\frac{r^{2}}{y}+2 y\right)\right] \\
r^{2}= & x^{2}+y^{2}, x=\left(u-c_{x}\right) / f_{x}, y=\left(v-c_{y}\right) / f_{y}
\end{aligned}
$$

$k_{1}$ and $k_{2}$ are the coefficients of radial distortions, and $p_{1}$ and $p_{2}$ are those of tangential ones. $c_{x}$ and $c_{y}$ are coordinates of the principal point. $f_{x}$ and $f_{y}$ are the focal lengths by the axes $x$ and $y . r$ is the distance of the ideal image point from the principal point. The values of $k_{1}, k_{2}, p_{1}, p_{2}, c_{x}$,

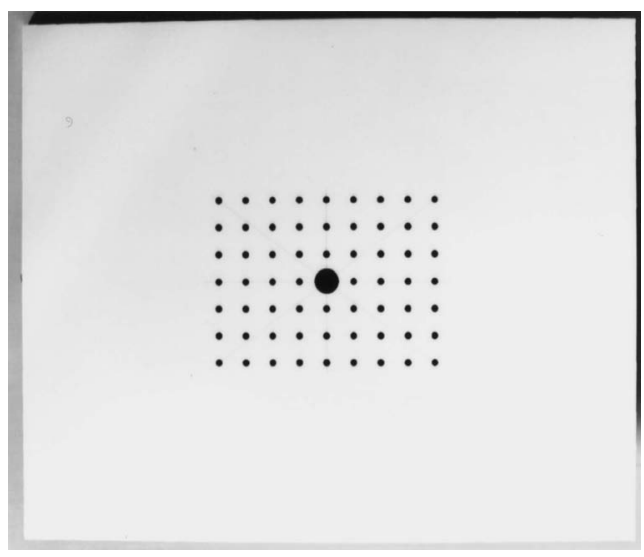

Fig. 1 Test chart. Diameters of small dots and the center dot are $7 \mathrm{~mm}$ and $25 \mathrm{~mm}$, respectively. Distance between each dot center is $28 \mathrm{~mm}$. The dot pattern is printed on the white board $65 \times 55$ $\mathrm{cm}$. $c_{y}, f_{x}$ and $f_{y}$ were estimated so that 63 lines connecting gravity points of 63 dots of the distorted image could be made straight by the least squares method (simulated annealing method ${ }^{16)}$. Furthermore, the software has been designed to perform correction from multiple chart images (maximum 10 images).

\subsection{Practice of the correction}

The images of the test charts were taken obliquely at various points of the lens field, and the image were reflected in the image display area of the 2D-Rugle software program (Medic Engineering, Japan). After the images were displayed in the display window of the DistRugle software program (Medic Engineering, Japan) for the correction, the coordinates of dots of those images were determined and the parameters such as focal lengths, and $k_{1}, k_{2}, p_{1}$ and $p_{2}$ were calculated (Fig. 2).

2.4. Examination in the reference camera and the surveillance camera

For the evaluation of the accuracy of the present method, the "ideal" (undistorted) image was created by parallel or rotating translation of the taken image by the perspective projection method ${ }^{14)}$. Camera parameters were obtained from 1 to 9 test chart images taken at

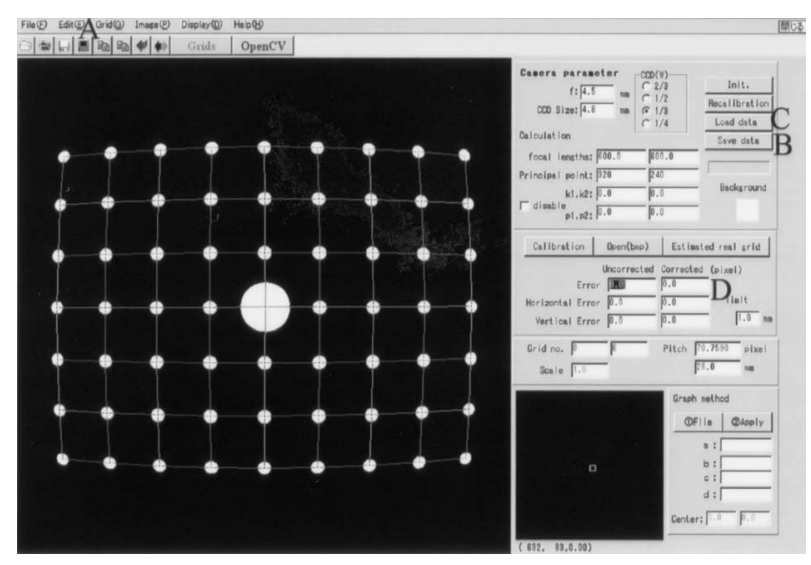

Fig. 2 Control window of the Dist-Rugle. A: key for grid setting by the binarization of chart images. B: key for saving the camera parameters. C: key for loading the camera parameters. The correction of the objective image is initiated by clicking this key. D: Error between the corrected grid image and the ideal image is displayed. 


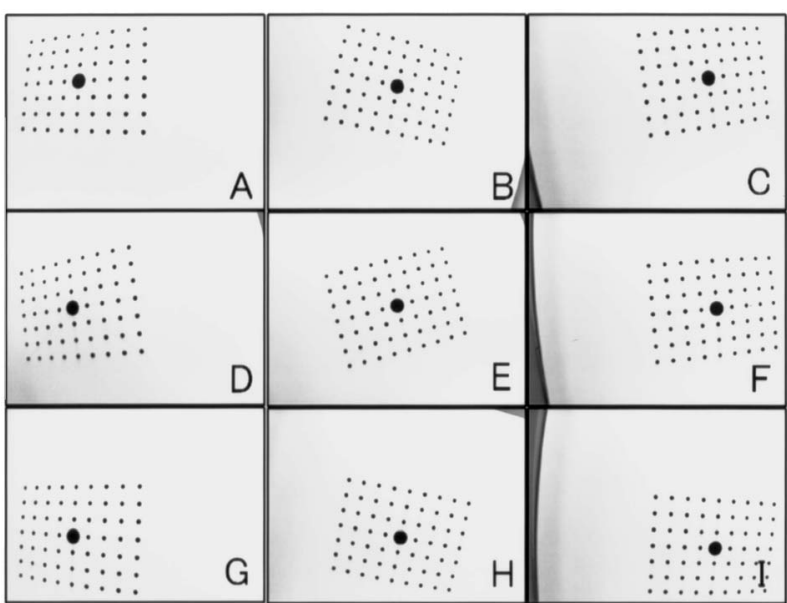

Fig. 3 Locations of test charts taken for camera calibrations. Calibrations were performed with 1 to 9 images of the test chart. An image of $E$ was used in a calibration from 1 image. Images of D and $\mathrm{F}$ were used to a calibration from 2 images. In the same way, images of $\mathrm{D}, \mathrm{E}$ and $\mathrm{F}$ to a calibration from 3 images, images of A, C, G and $I$ to a calibration from 4 images, images of A, C, E, G and I to a calibration from 5 images, images of $\mathrm{A}, \mathrm{B}, \mathrm{C}, \mathrm{G}, \mathrm{H}$ and $\mathrm{I}$ to a calibration from 6images, images of A, B, C, E, G, H and I to a calibration from 7 images, images of $\mathrm{A}, \mathrm{B}$, $\mathrm{C}, \mathrm{D}, \mathrm{F}, \mathrm{G}, \mathrm{H}$ and $\mathrm{I}$ to a calibration from $8 \mathrm{im}$ ages, images of A-I to a calibration from $9 \mathrm{im}$ ages.

various points of the lens field, as shown in Fig. 3. After one chart image was corrected for each parameter, the average of absolute distances (errors) of the corresponding dots between those on the corrected and ideal images was measured using Dist-Rugle. The images corrected by parameters obtained from each image taken at 20,30 and $40 \mathrm{~cm}$ from the object were also compared with each ideal image. After a facial image of a mannequin was taken at a distance from $40 \mathrm{~cm}$ by the surveillance camera, the image was corrected by the present method and was compared with a facial image taken at the same distance by the single lens reflex camera (Nikon, F3, lens $28 \mathrm{~mm}, \mathrm{~F} 2.8$ ).

\section{Other methods}

3.1. The graph method

The distortion graph shows levels $(\%)$ of

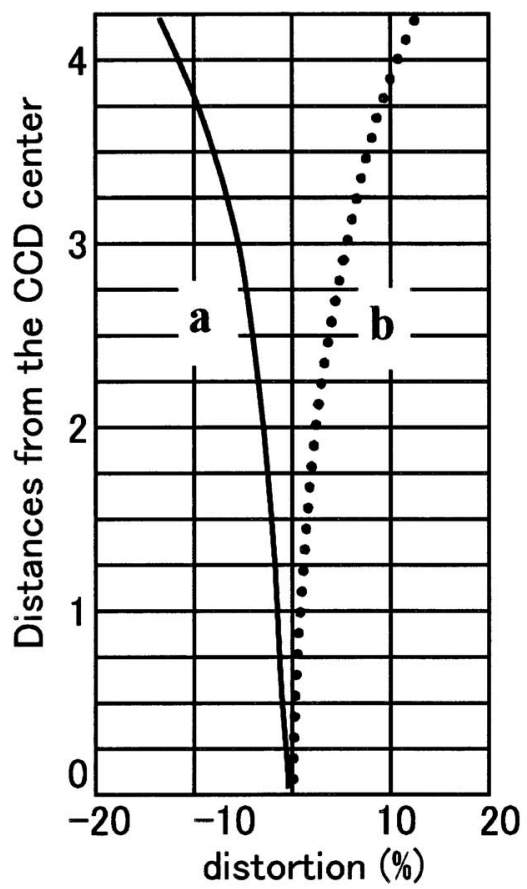

Fig. 4 Typical distortion graphs. Graph a: the barreled type image. Graph b: the bobbin type image.

distortion of each point from the center to the radial direction of the lens field. A typical distortion graph is shown in Fig. 4. The relationship between distortion $(D n(\%))$ and the distance from the center of the lens field to the actual focus location of the image $(Q n)$ can be expressed by the third regression equation

$$
D n=a \times Q n^{3}+b \times Q n^{2}+c \times Q n+d
$$

In which $a, b, c$ and $d$ are the constants. The relation between $D n$ and the distance from the center of the lens field to the ideal focus location $(P n)$ can be expressed as

$$
\begin{aligned}
D n & =\frac{(Q n-P n)}{P n} \times 100 \\
\text { whereas } \quad P n & =\frac{(Q n \times 100)}{(D n+100)}
\end{aligned}
$$

If $D n$ values corresponding to several $Q n$ values on the distortion graph are read and substituted in equation (1), the constants $a, b, c$, and $d$ can be calculated. Because $P n$ can be obtained from equation (2), all pixels of the distorted image can be moved to locations of calculated ideal coordinates. This correction program was coded 
using Dist-Rugle. We could obtain the distortion graph of the reference lens $(F=4.5 \mathrm{~mm})$, which had been provided by the camera maker. The image obtained by the graph method was compared with the ideal image as well as with the image obtained by the present method.

\subsection{The OpenCV method}

The camera calibration tool Open $\mathrm{CV}^{17)}$ was also compared with the present test chart method. This tool is programmed to automatically calculate distortion parameters from the video camera in real time while taking the checkerboard, which consisted of black and white lattices of $3 \times 3 \mathrm{~cm}$ (Fig. 5). Because the camera calibration tool could not recognize the output signal of the reference camera, the

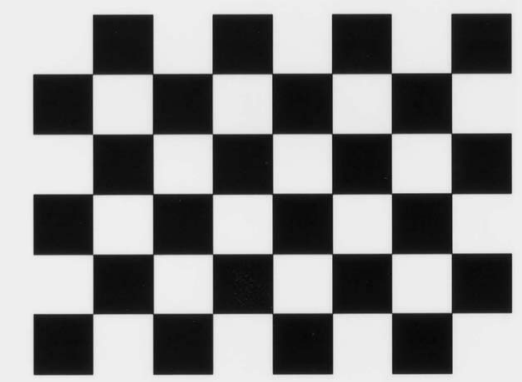

Fig. 5 Checkerboard pattern used in the OpenCV method. The pattern was printed on the white board. program was modified in order to calibrate with several still images of the checkerboard taken from various angles. The correction function using the distortion parameters obtained by the Open CV method was also included in DistRugle.

\section{Evaluation of the present method compared with other methods}

The average displacement errors of the 63 dots in images corrected by each method were examined. Errors observed between the image corrected by each method and the ideal image were illustrated in three-dimensional graphs.

\section{Results}

\section{Evaluation of the present method}

1.1. The reference camera

The results of calibrations with 1 to 9 chart images are shown in Table 1. Errors after the correction hardly changed and were approximately 0.200 pixels $(0.08 \mathrm{~mm})$, even if over 6 test chart images were used. The uncorrected image and the image corrected by the present method are shown in Fig. 6. Similar results were obtained using the camera with the $3.6 \mathrm{~mm}$ lens (data not shown). The relationships between the object distances and parameters are shown in Table 2. Better parameters could be obtained by taking images at a closer distance, because a bigger chart image covering the wide lens field was obtained.

\subsection{The surveillance camera}

The error was reduced from 20.76 pixels
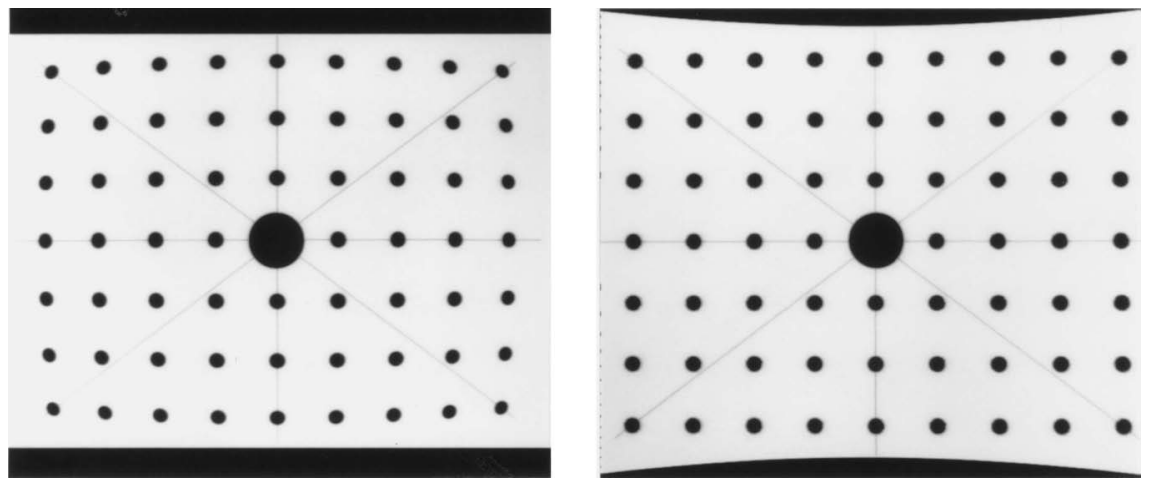

Fig. 6 Taken image (left) and image (right) corrected by the present method. 
Table 1 Results of calibrations with 1 to 9 images of dot chart.

\begin{tabular}{|c|c|c|c|c|c|c|c|c|c|c|}
\hline \multicolumn{2}{|c|}{ No. of images ${ }^{\mathrm{a})}$} & 1 & 2 & 3 & 4 & 5 & 6 & 7 & 8 & 9 \\
\hline \multirow{6}{*}{$\begin{array}{c}\text { Principal } \\
\text { points }^{\text {b) }}\end{array}$} & $\mathrm{x}-$ coordinate & 308.20 & 340.38 & 314.96 & 314.26 & 308.76 & 304.61 & 304.59 & 308.24 & 307.62 \\
\hline & $\mathrm{y}$-coordinate & 226.50 & 227.27 & 232.92 & 232.05 & 229.92 & 241.06 & 241.01 & 240.32 & 240.77 \\
\hline & $\mathrm{k}_{1}$ & 0.30389 & 0.28599 & 0.28168 & 0.27793 & 0.27826 & 0.28844 & 0.28778 & 0.28715 & 0.28749 \\
\hline & $\mathrm{k}_{2}$ & 0.02344 & 0.11736 & 0.16515 & 0.16078 & 0.14990 & 0.14232 & 0.14295 & 0.14759 & 0.14859 \\
\hline & $\mathrm{p}_{1}$ & 0.00000 & 0.00056 & 0.00342 & 0.00301 & 0.00111 & 0.00746 & 0.00744 & 0.00716 & 0.00741 \\
\hline & $\mathrm{p}_{2}$ & 0.00000 & 0.01693 & 0.00415 & 0.00385 & 0.00031 & -0.00162 & -0.00165 & 0.00047 & 0.00009 \\
\hline \multirow{2}{*}{ Errorc) } & & 8.49 & 8.83 & 8.90 & 8.69 & 8.78 & 9.03 & 9.03 & 9.02 & 9.02 \\
\hline & Corrected & 0.56 & 0.38 & 0.26 & 0.32 & 0.30 & 0.20 & 0.20 & 0.21 & 0.20 \\
\hline
\end{tabular}

Results were obtained with a camera $4.5 \mathrm{~mm}$ lens.

a) : Test chart was taken at each position shown in Fig. 3.

b) : Ideal coordinate is $(300,240)$

c) : Average of displacement errors between 63 coordinates of the ideal image and those of corrected image (unit: pixels).

Table 2 Relationship between the subject distances and camera parameters.

\begin{tabular}{ccrrr}
\hline \multicolumn{2}{c}{ Object distance $(\mathrm{cm})$} & \multicolumn{1}{l}{20} & \multicolumn{1}{l}{30} & \multicolumn{1}{c}{40} \\
\hline $\begin{array}{c}\text { Principal } \\
\text { points }\end{array}$ & $\mathrm{x}$-coordinate & 298.57 & 297.86 & 302.07 \\
& $\mathrm{y}$-coordinate & 237.61 & 239.77 & 256.14 \\
\hline \multirow{6}{*}{$\mathrm{k}_{1}$} & 0.29163 & 0.28717 & 0.27706 \\
& $\mathrm{k}_{2}$ & 0.13340 & 0.15714 & 0.27832 \\
& $\mathrm{p}_{1}$ & 0.00557 & 0.00711 & 0.01507 \\
& $\mathrm{p}_{2}$ & -0.00456 & -0.00560 & -0.00479 \\
\hline \multirow{2}{*}{ Error ${ }^{\mathrm{a})}$} & Uncorrected & 9.10 & 9.11 & 9.81 \\
& Corrected & 0.20 & 0.30 & 0.59
\end{tabular}

The corrections were carried by the chart, which was taken at the center of the lens field (position E of Fig. 3).

a) : Average of displacement errors between 63 coordinates of the ideal image and those of the corrected image (unit: pixels).

$(7.92 \mathrm{~mm})$ to 0.86 pixels $(0.33 \mathrm{~mm})$ after correction by the present method. As well as the result in the reference camera, errors after the correction hardly changed and became constant, even if over 6 test chart was used (Fig. 8). Figs. $7 \mathrm{~A}$ and $\mathrm{B}$ are facial images of a mannequin before and after the correction, respectively, and Fig. $7 \mathrm{C}$ is that taken by the single lens reflex camera. The facial image after the correction closely resembled that taken by the single lens reflex camera, especially in respect to the facial outline.

\section{Evaluation of the present method compared with other methods}

As a result of the examination of errors between dots of the ideal image and those of each correction method, the error value of the present method was the smallest, whereas that of the graph method was the greatest (Table 3). Errors between each dot of grid images before and after correction by were illustrated for each of the three methods in three-dimensional graphs (Fig. 9). Correction was very successful at the gamut of the lens field in the present method (Fig. 9-A), but the displacement errors in other two methods became larger at the periphery of the lens field (Fig. 9-B, C).

\section{Discussion}

We have previously examined some methods for the correction of distortion occurring in images taken by surveillance cameras $^{3-5)}$. Commercial software for distortion correction is designed to straighten curved lines in photographed images which were actually linear. Correction using the software, therefore, is heavily influenced by the location of linear reference materials in the field ${ }^{3)}$. In this study, we examined three methods for obtaining distortion parameters of two CCD cameras. The graph method using the distortion graph provided by the lens manufacturer seems reliable, especially with regard to radial distortion, because the graph is based on the actual measurements. However, the graph is not always available for users. Therefore, other camera calibration methods like the Open $\mathrm{CV}^{17)}$ are required in pratice $^{6-11)}$. Dist-Rugle can 


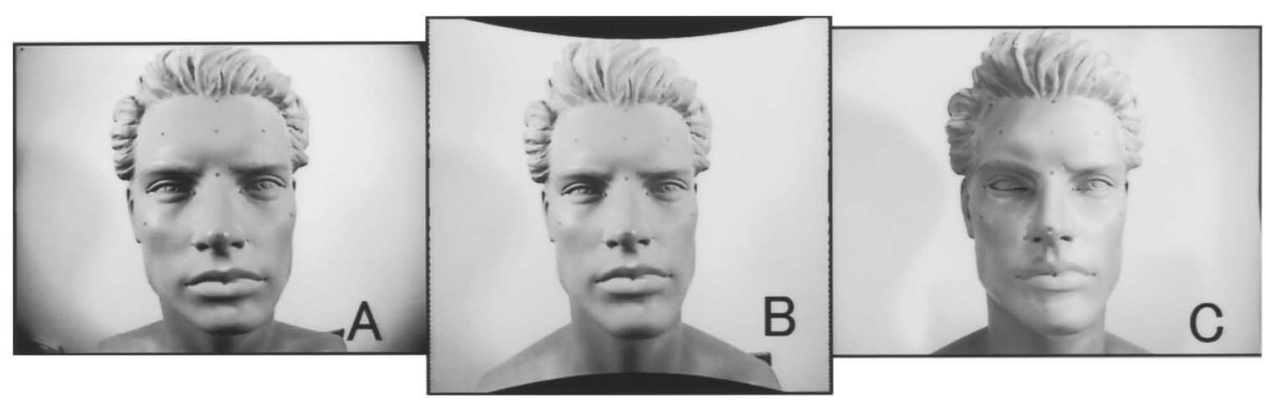

Fig. 7 Facial image of mannequin taken by the surveillance camera (A), facial image corrected by the present method (B) and facial image taken by the single lens reflex camera (C). All images were taken at a distance from $40 \mathrm{~cm}$.

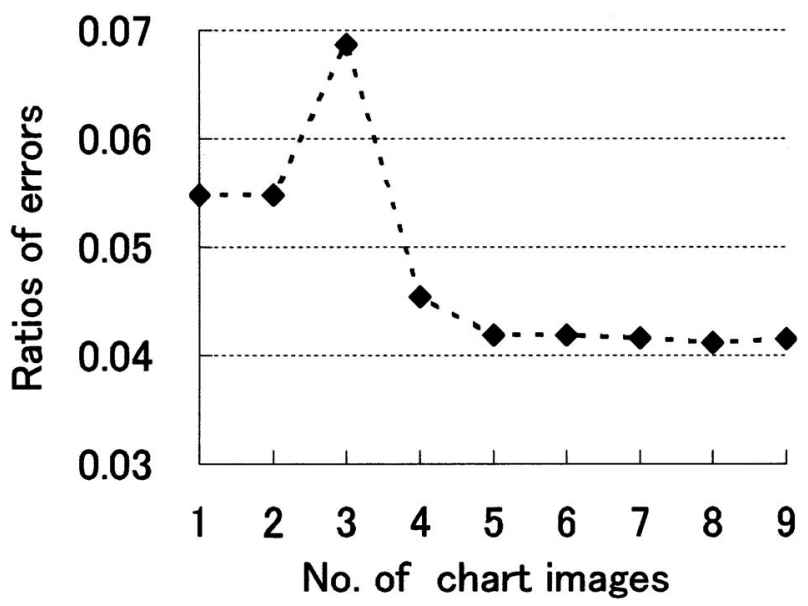

Fig. 8 Effect of correction and numbers of chart images. Vertical axis shows the value divided the error after correction by the error before correction.

automatically extract the gravities of dots on the chart by binary processing. Furthermore, this software is designed to make corrections by measuring distortions of several chart images located at various positions in the lens field. In this study, the use of six sheets (six positions) was necessary and sufficient to minimize errors (Table 1). Konuma ${ }^{14}$ and Murai et al. ${ }^{12}$ ) examined the relationship between various coefficients such as $k$ or $p$ and the error values in single lens reflex cameras, and reported that only coefficients $k_{1}$ and $k_{2}$, would be useful in distortion correction of such camera lenses. The present software can calculate the values of the tangential distortion coefficients $\left(p_{1}, p_{2}\right)$. The error in correction without using the tangential coefficients was about $0.11 \mathrm{~mm}$ bigger than that using the values from the surveillance cameras examined (data not shown). Therefore, calibration of the tangential distortion coefficients would be necessary in the surveillance camera, which had a wide angle lens. The error after correction of the reference camera was $0.08 \mathrm{~mm}$ and that of the surveillance cameras was $0.33 \mathrm{~mm}$. Because the distortion of peripheral areas of images taken by the surveillance camera may be extremely heavy, the results were attributed to inadequate correction of those areas. In fact, the average of the displacement errors in 28 dots of the outer area of the corrected test chart image was $0.26 \mathrm{~mm}$, whereas that of the 35 dots of the inner area of the image was $0.14 \mathrm{~mm}$ (data not shown). Therefore, distortions of several positions of the lens field should be taken into account in the inspection of face images. Moreover, although the corrected facial image of a mannequin showed good representation in terms of facial outlines compared with that taken by the single lens reflex camera, which had little distortion, detailed examination of comparing configuration of each facial area must be required. In addition, because images such as photographed faces were generally recorded with a videocassette recorder or a digital video recorder by the bank, the present method should be examined using preserved images. 
Table 3 Comparison between the present chart method and other methods.

\begin{tabular}{|c|c|c|c|c|c|c|c|c|c|c|c|c|}
\hline \multicolumn{2}{|c|}{ Methods } & \multicolumn{3}{|c|}{ Open CV } & \multicolumn{4}{|c|}{ Graph } & \multicolumn{4}{|c|}{ The present chart } \\
\hline Error $^{a)}$ & Average & SD & Min & $\begin{array}{l}\text { Max } \\
(\mathrm{mm})\end{array}$ & Average & $\mathrm{SD}$ & Min & $\underset{(\mathrm{mm})}{\operatorname{Max}}$ & Average & $\mathrm{SD}$ & Min & $\underset{(\mathrm{mm})}{\operatorname{Max}}$ \\
\hline Uncorrectec & 3.57 & - & - & - & 3.57 & - & - & - & 3.5 & - & - & - \\
\hline Corrected & $0.73^{b)}$ & 0.10 & 0.55 & 0.90 & $1.49^{\mathrm{b})}$ & 0.07 & 1.40 & 1.61 & $0.08^{c)}$ & 0.03 & 0.06 & 0.12 \\
\hline
\end{tabular}

a) : Errors occurred amongst the uncorrected image, the ideal image and images corrected by each correction method.

b) : Calibrations were carried out 10 times.

c) : Calibrations were carried out 10 times with 9 chart images (see Fig. 3).
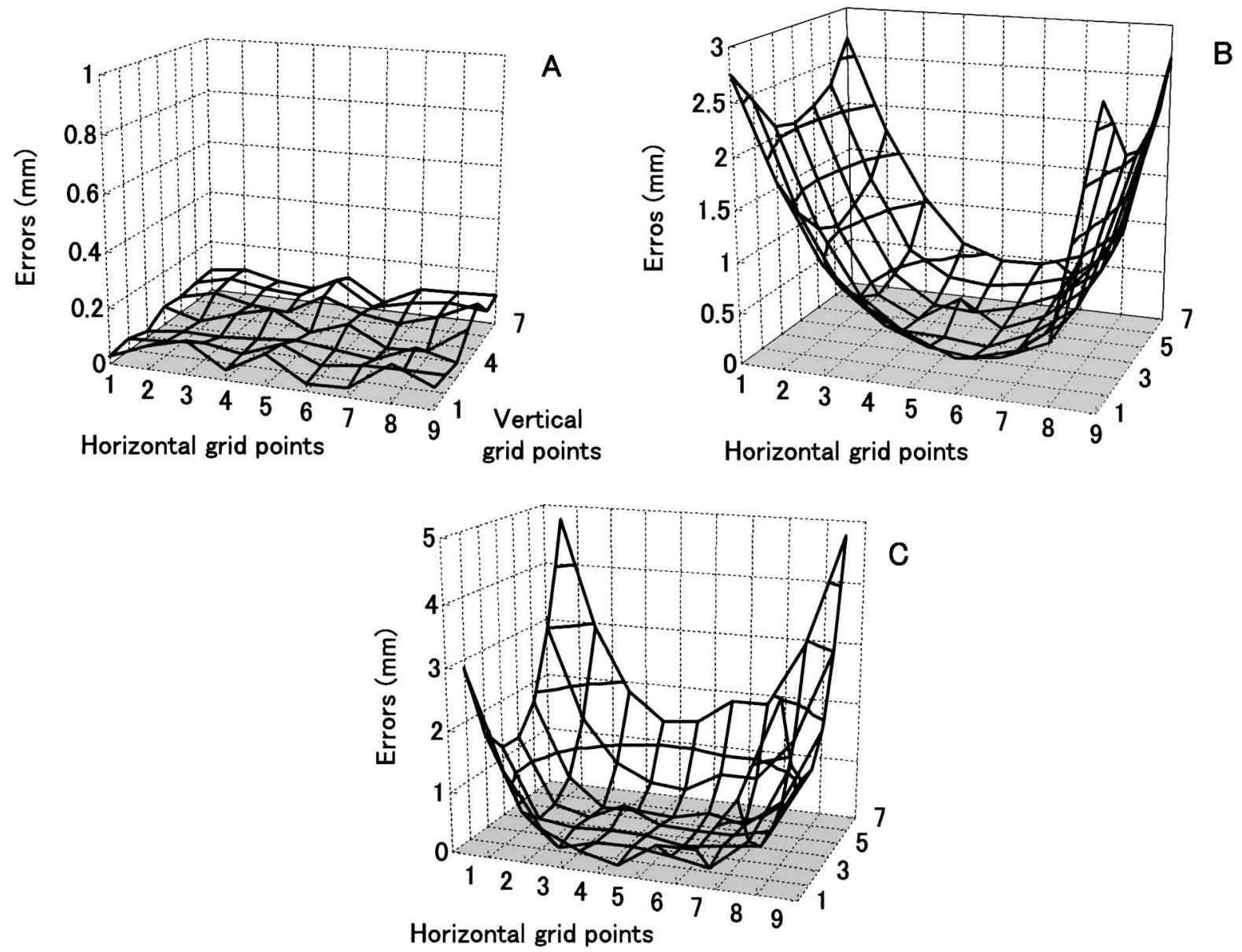

Fig. 9 Three-dimensional graph shown displacement errors of dots of chart images corrected by the present methods (A), the Open CV method (B) and the graph method (C). The basal plane (shaded zone) is presumed to be the ideal image plane.

The present chart method obtained better results than the other two methods. Because the center of the CCD in the camera and that of the lens often misaligned, errors of peripheral areas of the lens were field potentially increased in the graph method (Fig. 9-C). In addition, errors would result from the insufficient distortion data which would include those of only radial 
direction in the graph method. On the other hand, the camera calibration tool of Open CV was originally developed for digital video cameras, which have lenses almost free of distortion. Therefore, extreme distortion would be unmanageable.

The present method could deal with relatively heavy distortion, but can not always be applicable with all camera lenses. In the correction of distortion caused by a wide -angle lens, the examination using the test chart would be required and the error before and after the correction would need to be accurately evaluated.

\section{Acknowledgment}

The authors wish to thank Kita-Nippon Bank, Ltd. and Touhoku Sogo Security Services Co, Ltd. for the use of the surveillance camera.

\section{References}

1) Yoshino, M., Matsuda, H., Kubota, S., Imaizumi, K. and Miyasaka, S.: Assessment of computer-assisted comparison between 3D and 2D facial images. Jpn. J. Sci. Tech. Iden., 5 (1), 9-15 (2000).

2) Yoshino, M., Atsuchi, H., Matsuda, H., Kubota, S., Imaizumi, K., Tanijiri, H., Thomas, C. D. L. and Clement, J. G.: Evalution of outline matching in face-face superimposition images. Rep. Natl. Res. Inst. Police., 54(1), 1-6 (2001) (in Japanese) .

3) Biwasaka, H., Tokuta, T., Sasaki, Y., Tanijiri, H. and Miyasaka, S.: The computerized correction for optical distortion of facial images in face-face superimposition. Jpn. J. Sci. Tech. Iden., 9 (Suppl), 9 (2004) (in Japanese).

4) Biwasaka, H., Tokuta, T., Sasaki, T., Takagi, H., Miyasaka, S. and Aoki, Y.: The computerized correction for optical distortion of facial images in face-face superimposition (II). Jpn. J. Leg. Med., 59 (1), 69 (2005) (in Japanese).

5) Biwasaka, H., Tokuta, T., Sasaki, Y., Takagi, H., Tanijiri, H., Miyasaka, S. and
Aoki, Y.: The computerized correction for optical distortion of facial images in faceface superimposition (III). Jpn. J. Sci. Tech. Iden., 10 (Suppl), 33 (2005) (in Japanese).

6) Tsai, RY.: A versatile camera calibration technique for High-accuracy 3D machine vision metrology using off-the-shelf TV cameras and lenses. IEEE. J. Robo. Autom., 3, 323-344 (August 1987).

7) Zhang, Z.: Flexible camera calibration by viewing a plane from unknown orientations. Proc. of the Seventh IEEE Int. Conf. Comput. Vis., 1, 666-673 (1999).

8) Prescott, B. and Mclean, G.F.: Line-based correction of radial lens distortion. Graphical Models and Image Processing., 59 (1), 39-47. (1997).

9) Shah, S and Aggarwal, J. K.: Intrinsic parameter calibration procedure for a (highdistortion ) fish-eye lens camera with distortion model and accuracy estimation. Pattern Recognit., 29(11), 1775-1788 (1996).

10) Ahmed, M. T. and Farag, A. A.: Differential methods for nonmetric calibration of camera lens distortion. Proc. IEEE Comput. Soc. Conf. Comput. Vis. Pattern Recognit., 2, 477-482 (2001).

11) Guan, H., Shiraishi, K., Watanabe, K., Fukuoka, H. and Ohashi, K.: Digital image correction method for digital camera. Ricoh Technical report. 31, 103-110 (December, 2005).

12) Murai, S., Matsuoka R., Okuda T.: Comparison of accuracy amongst adjustment methods. J. Jpn. Soc. Photogrammetry., 23(2), 4-11 (1984).

13) Hattri, S.: Bundle adjustments in nontopographic photogammetry. J. Jpn. Soc. Photogrammetry., 27(2), 16-22 (1988) (in Japanese).

14) Konuma H.: Accuracy of analytical real space measurement from a single image by non-metric camera. Rep. Natl. Res. Inst. Police., 52 (2), 1-6 (1999) (in Japanese).

15) Dai, S. and Ji, Q.: A new technique for camera self-calibration. Proc. IEEE Int. 
Conf. Rob. Autom., 3, 2165-2170 (2001).

16) Tanijiri, T.: Practice correction method for the distortion of the surveillance camera lens. Image Labo., 17(7) 1-7 (2006). (in
Japanese).

17 ) http: / / www.sourceforge.net / projects / opencvlibrary 